\title{
Cancer-associated Fibroblast-derived Spondin-2 Promotes Motility of Gastric Cancer Cells
}

\author{
SHOTARO KURAMITSU ${ }^{1 *}$, TAKAAKI MASUDA ${ }^{1 *}$, QINGJIANG HU ${ }^{1,2}$, TARO TOBO ${ }^{3}$, \\ MASAKAZU YASHIRO ${ }^{4}$, ATSUSHI FUJII ${ }^{1}$, AKIHIRO KITAGAWA ${ }^{1}$, TADASHI ABE ${ }^{1}$, \\ HAJIME OTSU $^{1}$, SHUHEI ITO ${ }^{1}$, EIJI OKI ${ }^{2}$, MASAKI MORI ${ }^{2}$ and KOSHI MIMORI ${ }^{1}$ \\ ${ }^{I}$ Department of Surgery, Kyushu University Beppu Hospital, Beppu, Japan; \\ ${ }^{2}$ Department of Surgery and Science, Kyushu University Hospital, Fukuoka, Japan; \\ ${ }^{3}$ Department of Clinical Laboratory Medicine, Kyushu University Beppu Hospital, Beppu, Japan; \\ ${ }^{4}$ Department of Gastroenterological Surgery, Osaka City University Graduate School of Medicine, Osaka, Japan
}

\begin{abstract}
Background/Aim: Peritoneal dissemination (PD) occurs frequently in gastric cancer $(G C)$ and is fatal. The interactions between tumor cells and stromal cells are critical for cancer progression. Our aim was to identify a novel PDassociated gene derived from stromal cells in GC. Materials and Methods: Among the candidate PD-associated genes identified in our previous study, we focused on spondin-2 (SPON2), an extracellular matrix-secreted protein. Clinicopathological and prognostic analyses of SPON2 mRNA expression were performed using GC datasets. Localization of SPON2 expression was assessed by immunohistochemistry. In vitro migration assay and immunofluorescence staining were also conducted using GC cell lines. Results: SPON2 was expressed in and secreted from cancer-associated fibroblasts in GC. High expression of SPON2 in tumor tissues was correlated with PD, tumor size and poor prognosis in GC. The motility of GC cells was increased by treatment with a SPON2 recombinant protein in vitro. Conclusion: Cancer-associated fibroblast-derived $S P O N 2$ may promote PD, in part, by facilitating GC cell motility and serve as a predictive marker for $P D$ in $G C$.
\end{abstract}

This article is freely accessible online.

*These Authors contributed equally to this study.

Correspondence to: Koshi Mimori, MD, Ph.D., Department of Surgery, Kyushu University Beppu Hospital, 4546 Tsurumihara, Beppu 874-0838, Japan. Tel: +81 977271650, Fax: +81 977271651, e-mail:kmimori@beppu.kyushu-u.ac.jp

Key Words: Gastric cancer, SPON2, peritoneal dissemination, cancer-associated fibroblasts.
Gastric cancer (GC) is a common malignancy and a leading cause of cancer-related death worldwide, especially in East Asia (1). The malignant potential of GC is characterized by peritoneal dissemination (PD), defined as disseminating tumor cells throughout the peritoneal cavity. In a clinical study, PD is diagnosed in $14 \%$ of GC patients at the initial examination, and the survival time of such patients is only 1 year, although multimodal therapies have improved the clinical outcome of GC patients with PD (2-7). Therefore, identification of biomarkers and therapeutic targets for PD is warranted to improve the prognosis of GC patients.

Recent in silico analyses using large-scale public datasets such as The Cancer Genome Atlas (TCGA) have contributed to identifying candidate driver genes responsible for metastasis or poor prognosis. We previously identified DDR2, ARL4C and FGFR1 as PD-related driver genes of GC cells (8-10). However, recent cancer research has shed light on the interactions between cancer cells and various stromal cells, including fibroblasts, endothelial cells and immune cells in the tumor microenvironment (TME) (11-15).

The TME is believed to promote cancer cell invasion, followed by metastasis. We recently reported that KIF15, which is expressed in host inflammatory monocytes, facilitates the proliferation of tumor cells in hepatocellular carcinoma (16). Thus, we hypothesized that stromal cells in the TME contribute to PD of cancer cells in GC. In this study, we demonstrated that spondin-2 (SPON2), an extracellular matrix-secreted protein, is a novel PDassociated gene derived from stromal cells in the TME and determined its clinical and biological significance in GC.

\section{Materials and Methods}

TCGA dataset. We obtained mRNA expression and clinical data from 406 GC patients from the Broad Institute's Firehose 
(http://gdac.broadinstitute.org/runs/stddata_2015_06_01/data/ST $\mathrm{AD} / 20150601 /$ ). The mRNA expression data (FPKM, raw count) from 406 tumor tissues and 31 normal tissues were subjected to quantile normalization. Clinical data were available from 375 patients.

GSE15459 dataset. GSE15459 dataset was downloaded from the Gene Expression Omnibus (https://www.ncbi.nlm.nih.gov/geo/). This dataset consists of mRNA expression and clinical data from $198 \mathrm{GC}$ patients in Singapore but contains no mRNA expression data from normal tissues. The mRNA expression data were subjected to quantile normalization. Because PD data, as well as clinical data, were available from 174 of these patients, we used the data from these patients to identify PD-associated genes. Of the 174 patients, there were 32 with PD (peritoneal nodules or positive cytology) and 142 without PD.

Beppu dataset. Cancer tissues were obtained from $170 \mathrm{GC}$ patients who underwent gastrectomy at the Kyushu University Beppu Hospital and affiliated hospitals from 1995 to 2009 after providing written informed consent. Of these patients, 47 were excluded because of either loss to follow-up or poor sample quality. Thus, we enrolled $123 \mathrm{GC}$ patients in our survival analysis. The median follow-up period of these patients were 586 days. Paired tumor and normal tissues were obtained from 98 GC patients. All data associated with the samples including age, sex, tumor size, depth of tumor invasion and lymph node metastasis were obtained from clinical records. We treated the patients according to the Japanese Gastric Cancer Treatment Guidelines. No patients received neoadjuvant systemic therapy.

The tumor and normal tissues were placed in RNAlater (Takara Bio Inc, Shiga, JAPAN), frozen in liquid nitrogen and stored at $-80^{\circ} \mathrm{C}$. This study was approved by the Ethics and Indications Committee of Kyushu University (\#2020-302).

Cell culture. The human GC cell lines AGS, KE-39, MKN1, MKN7, MKN28, MKN45, MKN74 and NUGC3 and the human fibroblast cell line KMST6 were purchased from the Japanese Collection of Research Bioresources Cell Bank, National Institutes of Biomedical Innovation, Health and Nutrition (Osaka, Japan). The human GC cell lines NUGC4, SH10TC and KLN205 were purchased from RIKEN BioResource Center (Tsukuba, Japan). The human leukemia cell lines Jurkat, Raji and THP1 were purchased from the Cell Resource Center for Biomedical Research, Institute of Development, Aging and Cancer, Tohoku University (Miyagi, Japan). Two human GC-associated fibroblast (CAF) lines (CAF-100 and CAF-102) derived from surgical gastric tissue were established at Osaka City University as described previously (17).

AGS, KE-39, MKN1, MKN7, MKN28, MKN45, MKN74, NUGC3, NUGC4, SH10TC, KLN205, Jurkat, Raji and THP1 cells were maintained in Roswell Park Memorial Institute (RPMI) 1640 medium, and KMST6 cells were maintained in Dulbecco's Modified Eagle's Medium. All media contained 10\% fetal bovine serum, 100 $\mathrm{U} / \mathrm{ml}$ penicillin and $100 \mathrm{U} / \mathrm{ml}$ streptomycin sulfate. CAF-100 and CAF-102 were maintained in Dulbecco's Modified Eagle Medium containing $10 \%$ fetal bovine serum, $100 \mathrm{U} / \mathrm{ml}$ penicillin, $100 \mathrm{U} / \mathrm{ml}$ streptomycin sulfate and $10 \%$ glucose. All cells were cultured in a humidified $5 \% \mathrm{CO}_{2}$ incubator at $37^{\circ} \mathrm{C}$.

Total RNA extraction and reverse transcription-quantitative polymerase chain reaction ( $R T-q P C R)$. Total RNA was extracted from cell lines and tissues using ISOGEN (Nippon Gene, Tokyo, Japan) according to the manufacturer's instructions. $8 \mu \mathrm{g}$ total RNA was reverse transcribed using M-MLV reverse transcriptase (Invitrogen, Carlsbad, CA, USA). qPCR analysis of SPON2 and 18S was performed using LightCycler FastStart DNA Master SYBR Green I (Roche Diagnostics, Indianapolis, IN, USA) as described previously (18). The mRNA level of SPON2 was normalized to that of $18 \mathrm{~S}$, as an internal control, and expressed relative to the level of the cDNA from human universal reference total RNA (Takara Bio Inc, Shiga, Japan).

Immunohistochemical analysis. Immunohistochemical analysis of SPON2 and $\alpha$ SMA was performed using formalin-fixed paraffinembedded surgical sections obtained from patients with GC, as described previously (19). A primary rabbit polyclonal antibody against SPON2 (HPA040170; Sigma Aldrich, St. Louis, MO, USA) was used at a dilution of 1:100, and a mouse monoclonal antibody against $\alpha$ SMA (MA5-11544, Thermo Fisher Scientific, Waltham, MA, USA) was used at a dilution of 1:100. Histological analysis was performed independently by an experienced research pathologist (TT) at Kyushu University Beppu Hospital.

Western blot analysis. Western blot analysis was performed as previously described (8). Cell lysates were mixed with a $10 \times$ volume of $10 \%$ trichloroacetic acid in acetone, incubated for $1 \mathrm{~h}$ at $-20^{\circ} \mathrm{C}$, and centrifuged at $14,000 \mathrm{rpm}$ for $5 \mathrm{~min}$ at $4^{\circ} \mathrm{C}$. Precipitates were washed in ice-cold acetone, air-dried at room temperature, and dissolved in lysis buffer. The following specific antibodies were used: primary rabbit polyclonal antibody against SPON2 (HPA040170; Sigma Aldrich) at a dilution of 1:250 and primary mouse polyclonal antibody against $\beta$-actin (Santa Cruz Biotechnology, Santa Cruz, CA, USA) at a dilution of 1:10,000. The protein expression of SPON2 was normalized to that of $\beta$-actin.

Gene set enrichment analysis (GSEA). The associations between SPON2 expression and previously defined gene sets were analyzed by GSEA using GC expression profiles from TCGA dataset. Biologically defined gene sets were obtained from the Molecular Signatures Database v5.2 (http://software.broadinstitute.org/gsea/ msigdb/index.jsp).

SPON2 recombinant protein ( $r S P O N 2)$. SPON2 recombinant protein was purchased from Sigma-Aldrich (HPA043890).

Wound healing migration assay. For the wound healing migration assay, $3 \times 10^{5}$ cells were plated per well of a 6-well plate and treated with rSPON2 and control $\mathrm{ddH}_{2} \mathrm{O}$ in triplicate. After the cells reached $80-90 \%$ confluence, vertical scratches were generated in the cell layer using a 1,000 $\mu$ l pipette tip. Photographs of the wound area were taken at 0,96 and $196 \mathrm{~h}$ after scratch formation to calculate the cell migration rate.

Immunofluorescence analysis. The actin stain 488 phalloidin (Cytoskeleton, Denver, CO, USA) was used to stain actin filaments in AGS and KE39 cells treated with rSPON2, and fluorescent images were observed using a fluorescence microscope (BZ-X700, Keyence, Osaka, JAPAN). We counted the number of cells containing lamellipodia.

Statistical analysis. GC was staged in all patients according to the $7^{\text {th }}$ edition of the Union for International Cancer Control TNM 
A

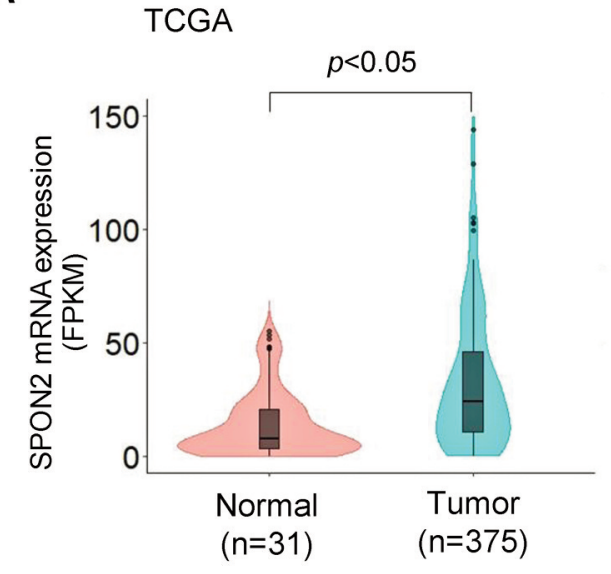

B

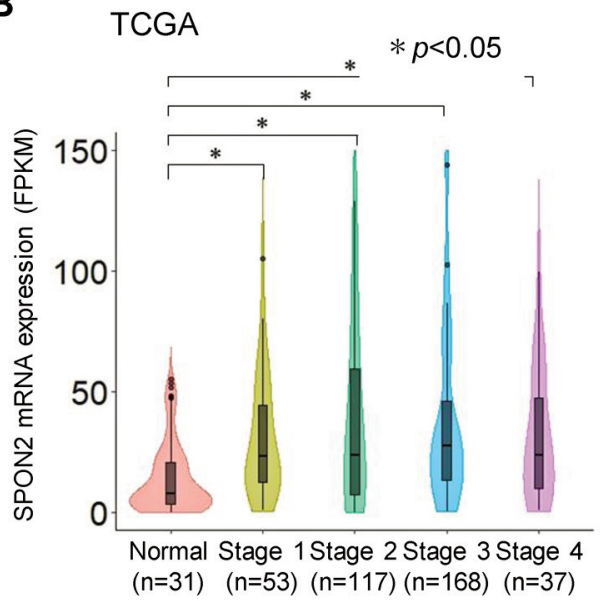

Beppu

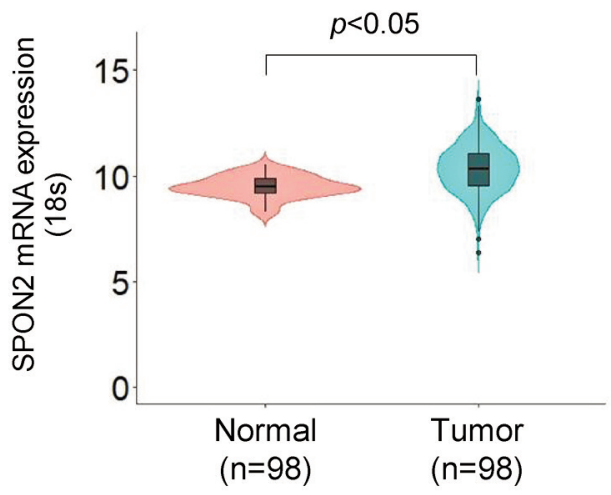

C

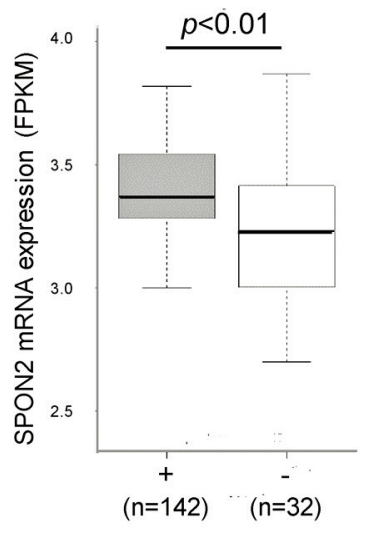

Peritoneal dissemination
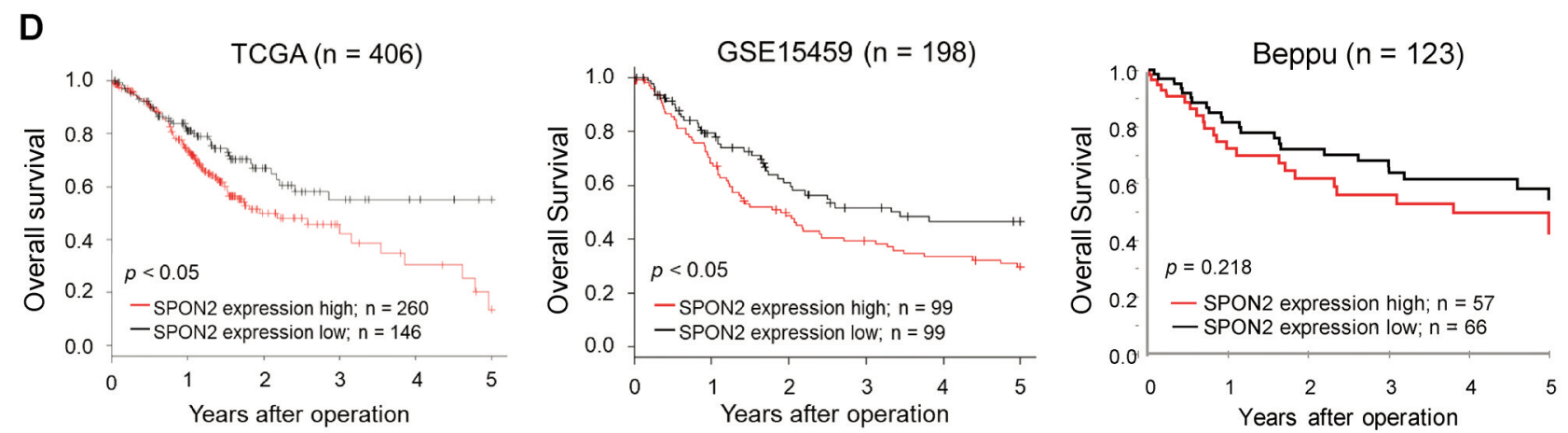

Figure 1. Spondin2 was identified as a PD-associated candidate gene in gastric cancer. (A) SPON2 mRNA expression in tumor and normal tissues from gastric cancer patients. Left: TCGA dataset. Right: Beppu dataset. (B) SPON2 mRNA expression in tumor tissues in gastric cancer patients according to TNM stage. Left: TCGA dataset. Right: Beppu dataset. (C) Comparison of SPON2 mRNA expression in GC patients between with and without PD in GSE15459 dataset. (D) Kaplan-Meier curves of overall survival in gastric cancer patients according to SPON2 mRNA expression. Left: TCGA dataset. Middle: GSE15459 dataset. Right: Beppu dataset. 
classification. For the clinical analyses, the patients were divided into two groups based on the SPON2 expression level using the minimum $p$-value approach to identify the optimal SPON2 expression value for stratifying the patients to evaluate survival (20). Associations between the variables were assessed using Student's $t$-test or Fisher's exact test. The degree of linearity was estimated using Pearson's correlation coefficient. OS was estimated using the Kaplan-Meier method, and survival curves were compared using the log-rank test. A two-sided $p<0.05$ was considered significant. Data analyses were performed using R software v3.1.1 (The R Foundation) and JMP Pro 15 software (SAS Institute, Cary, NC, USA).

\section{Results}

SPON2 is a candidate PD-associated gene in GC. In our previous study, we identified candidate GC PD-associated genes that satisfied the following three criteria: 1) overexpression in GC tissues with PD compared to GC tissues without PD in the GSE15459 dataset, 2) overexpression in GC tissues compared to normal tissues in TCGA datasets, and 3) high gene expression identified as a poor prognostic factor in GC patients according to a TCGA dataset (9). SPON2 was among these candidate genes. SPON2 encodes an extracellular matrix-secreted protein that has multiple biological functions in processes including neuronal development and innate immune response (21). SPON2 was reported to promote invasion, which is important for PD (22).

Figure 1 shows the verification of SPON2 as a PDassociated gene in GC. The expression of SPON2 mRNA was significantly higher in GC tissues than in normal tissues in TCGA and Beppu datasets (Student's $t$-test, $p<0.05$ ) (Figure 1A). However, there was no statistical difference in SPON2 mRNA expression according to TNM stage in those datasets (Figure 1B). Notably, the expression of SPON2 mRNA was higher in GC tissues with PD than in those without PD in the GSE15459 dataset $(p<0.01)$ (Figure 1C).

The prognostic significance of SPON2 mRNA expression in these three GC datasets is shown in Figure 1D. The trends indicated that high SPON2 expression in GC tissues was associated with a poorer prognosis. A statistically significant difference in OS according to SPON2 expression was noted in TCGA and GSE15459 datasets (log-tank test, $p<0.05)$.

SPON2 is expressed mainly in CAFs in GC. We measured SPON2 mRNA expression by RT-qPCR in various cancer cell lines, including fibroblasts, lymphoblasts, muscle cells and malignant cells (fibroblast lines: CAF-100, CAF102 and KMST6; GC cell lines: MKN1, MKN7, MKN28, MKN45, MKN74, NUGC3, NUGC4, AGS, SH10TC and KLN205; leukemia and lymphoma cell lines: Jurkat, Raji and THP-1). Interestingly, SPON2 mRNA expression was highest in the two CAF cell lines among all cell lines evaluated (Figure $2 \mathrm{~A}$, Student's $t$-test, $p<0.05)$.
Table I. SPON2 mRNA expression and clinicopathological factors in Beppu dataset $(n=123)$.

\begin{tabular}{|c|c|c|c|}
\hline \multirow[t]{2}{*}{ Factors } & $\begin{array}{c}\text { High SPON2 } \\
\text { mRNA } \\
\text { expression } \\
(n=57)\end{array}$ & $\begin{array}{c}\text { Low SPON2 } \\
\text { mRNA } \\
\text { expression } \\
(n=66)\end{array}$ & \multirow[t]{2}{*}{$p$-Value } \\
\hline & Number $(\%)$ & Number (\%) & \\
\hline Age (years) & & & 0.852 \\
\hline$<65$ & $21(36.8)$ & $23(34.8)$ & \\
\hline$\geq 5$ & $36(63.2)$ & $43(65.2)$ & \\
\hline Gender & & & 0.708 \\
\hline Male & $36(63.2)$ & $44(66.7)$ & \\
\hline Female & $21(36.8)$ & $22(33.3)$ & \\
\hline Histology & & & 0.857 \\
\hline Intestinal type & $26(45.6)$ & $32(48.5)$ & \\
\hline Diffuse type & $31(54.4)$ & $34(51.5)$ & \\
\hline Tumor size $(\mathrm{cm})$ & & & $0.016^{* *}$ \\
\hline$\leq 3$ & $7(12.3)$ & $21(31.8)$ & \\
\hline$<3$ & $49(86.0)$ & $44(66.7)$ & \\
\hline Unknown & $1(1.7)$ & $1(1.5)$ & \\
\hline Depth of invasion & & & \\
\hline (muscularis propria invasion) & & & 0.053 \\
\hline Absent & $8(14.0)$ & $19(28.8)$ & \\
\hline Present & $49(86.0)$ & $47(71.2)$ & \\
\hline Lymphatic involvement & & & 1.000 \\
\hline Absent & $21(36.8)$ & $25(37.9)$ & \\
\hline Present & $36(63.2)$ & $41(62.1)$ & \\
\hline Vascular involvement & & & 0.844 \\
\hline Absent & $41(71.9)$ & $46(69.7)$ & \\
\hline Present & $16(28.1)$ & $20(30.3)$ & \\
\hline Lymph node metastasis & & & 0.338 \\
\hline Absent & $16(28.1)$ & $25(37.9)$ & \\
\hline Present & $41(71.9)$ & $41(62.1)$ & \\
\hline Peritoneal dissemination & & & 0.282 \\
\hline Absent & $48(84.2)$ & $60(90.9)$ & \\
\hline Present & $9(15.8)$ & $6(9.1)$ & \\
\hline Distant metastasis* & & & 0.120 \\
\hline Absent & $41(71.9)$ & $56(84.8)$ & \\
\hline Present & $16(28.1)$ & $10(15.2)$ & \\
\hline
\end{tabular}

*including peritoneal dissemination; **statistically significant.

Next, we analyzed the correlations between the mRNA level of SPON2 and those of CAF markers (smooth muscle alphaactin 2, fibroblast activation protein alpha, periostin, desmin, vimentin, tenascin $\mathrm{C}$, platelet-derived growth factor subunit $\mathrm{B}$ and S100 calcium binding protein A4) using a TCGA dataset (12). SPON2 mRNA expression was positively correlated with the mRNA expression of all CAF markers (Figure 2B, Spearman's correlation coefficient, $\mathrm{R}=0.24-0.68, p<0.01)$.

Of note, immunohistochemical analysis indicated that SPON2 was expressed mainly in aSMA-positive fibroblasts, namely CAFs, in GC tissue (12) (Figure 2C). Furthermore, secreted SPON2 protein was detected in the supernatants of the CAF cultures (Figure 2D). Taken together, our results 
A

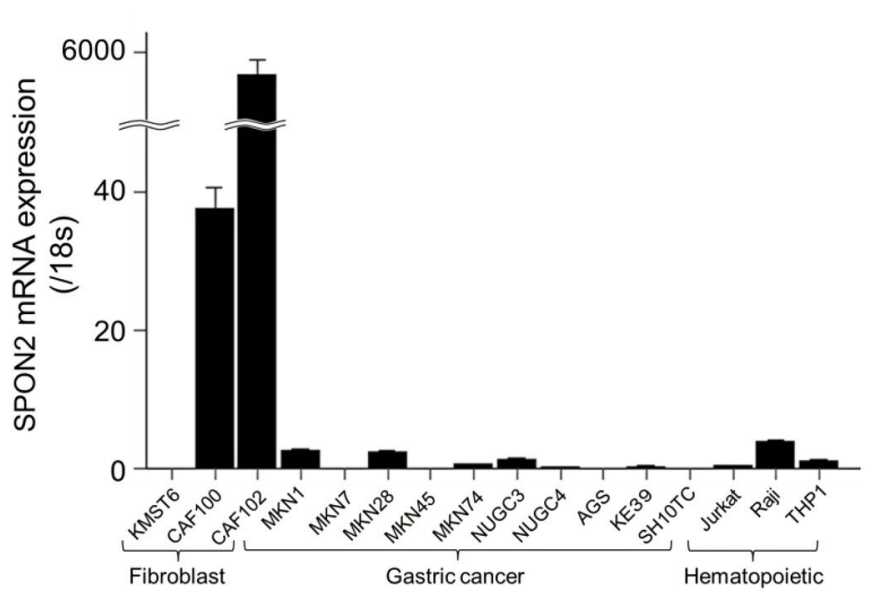

C
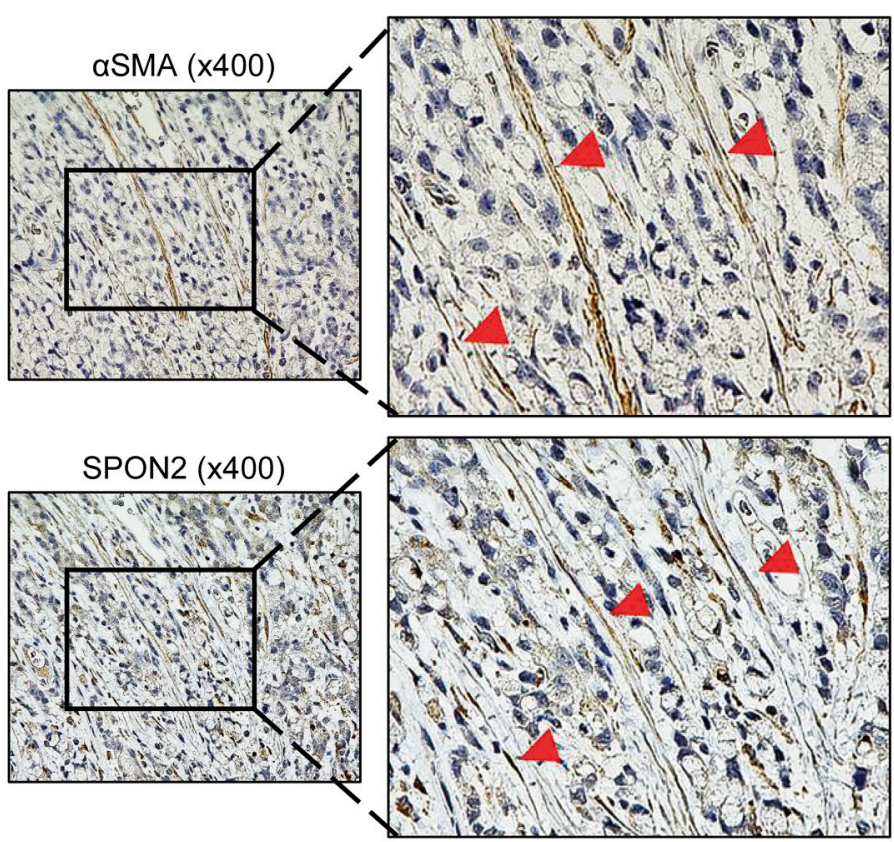

B
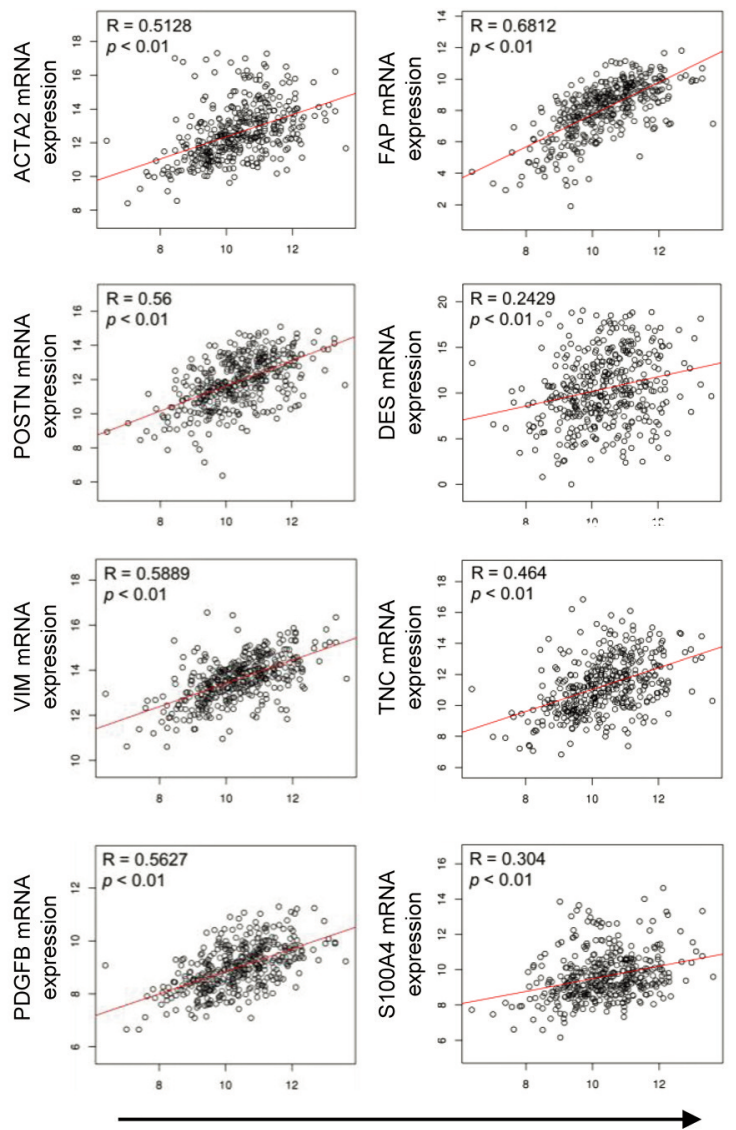

SPON2 mRNA expression

D

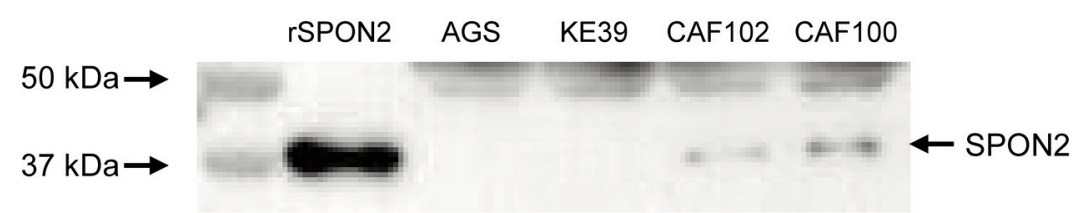

Figure 2. SPON2 is expressed in cancer-associated fibroblasts in gastric cancer tissues. (A) Comparison of SPON2 mRNA expression among fibroblast, gastric cancer and hematopoietic cell lines. SPON2 mRNA was highly expressed in cancer-associated fibroblasts. (B) Correlations between mRNA levels of SPON2 and those of cancer-associated fibroblast markers in gastric cancer patients from the TCGA dataset. The cancerassociated fibroblasts markers were smooth muscle alpha-actin 2, fibroblast activation protein alpha, periostin, desmin, vimentin, tenascin C, platelet-derived growth factor subunit B and S100 calcium binding protein A4. (C) Immunohistochemical staining of SPON2 in representative gastric cancer tissues. Original magnification, $\times 200$ and $\times 400$. (D) SPON2 protein expression in the supernatant of cancer-associated fibroblast lines by western blotting. 
A KEGG_TGF_BETA_SIGNALING_PATHWAY

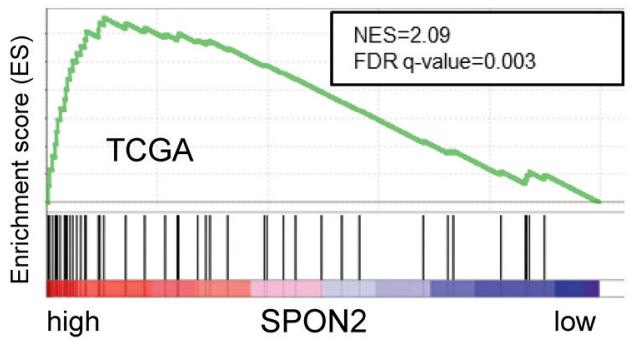

HALLMARK_EPITHELIAL_MESENCHYMAL_TRANSITION

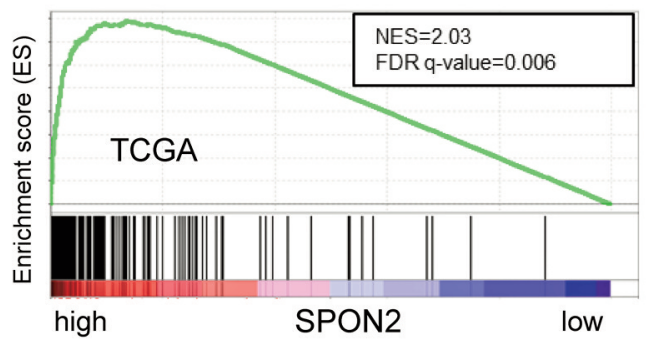

B
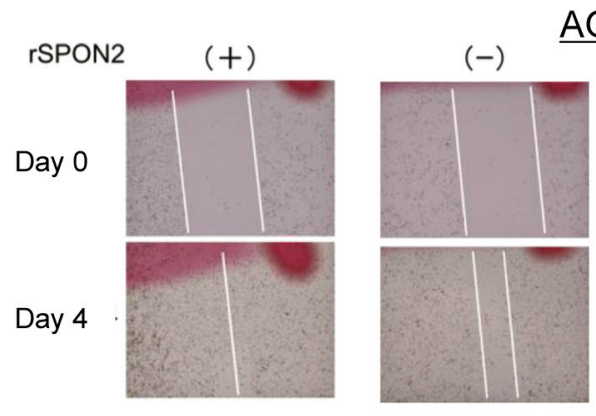

AGS

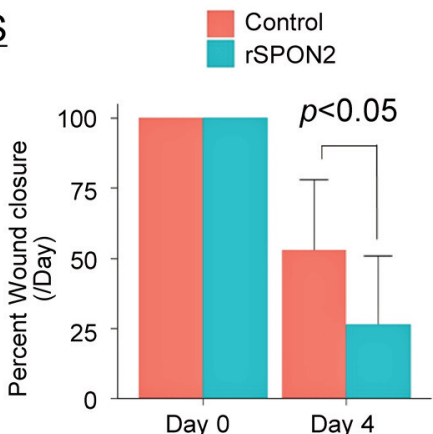

\section{KE39}

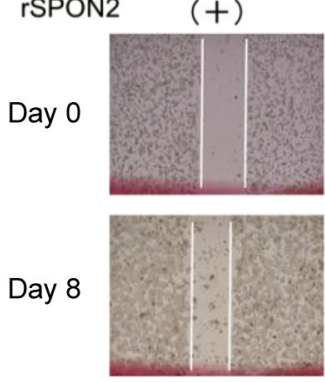
$(-)$

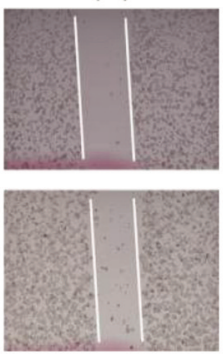

궁 $p<0.05$

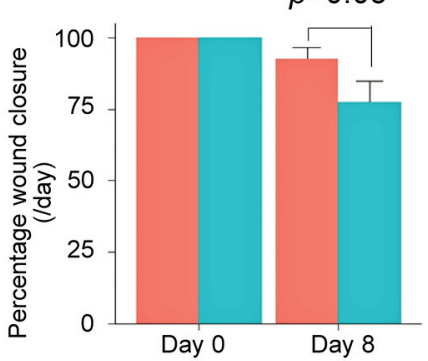

Figure 3. Continued

provide evidence that SPON2 is expressed in and secreted from CAFs in GC tissues.

SPON2 mRNA expression has clinicopathological significance in GC patients. As shown in Table I, high SPON2 mRNA expression in GC tissues was positively associated with tumor size (Fisher's exact test, $p<0.05$ ). Although the rate of PD positivity was higher in GC tissues with high SPON2 expression than in those with low expression ( $15.8 \%$ vs. 9.1\%), the difference was not significant, which may be explained by the small number of GC patients with PD in Beppu dataset.

SPON2 promotes the motility of GC cells in vitro. To investigate the effect of SPON2 expression on PD, we performed GSEA using TCGA datasets. GSEA showed that high SPON2 expression was positively correlated with transforming growth factor $\beta$ (TGF- $\beta$ ) and epithelialmesenchymal transition (EMT) gene sets (Figure 3A). An increased level of TGF- $\beta$ in the TME can induce tissueresident fibroblasts to acquire a CAF phenotype, such as a high migration ability, which is associated with up-regulation of $\alpha$-SMA (23). EMT is a critical switch for tumor cell invasiveness, leading to PD (24). Therefore, we focused on the role of SPON2 in migration in GC and performed in vitro experiments to elucidate these mechanisms. We performed a wound healing migration assay using rSPON2 in AGS and KE39 cells. rSPON2 enhanced the migration capacity of both AGS and KE39 cells (Student's $t$-test, $p<0.05$, Figure 3B).

A line of evidence suggests that SPON2 expression induces actin cytoskeleton remodeling, such as pseudopodium formation, a process that is important for the motility of tumor cells (25). To examine the role of SPON2 expression in GC 
C
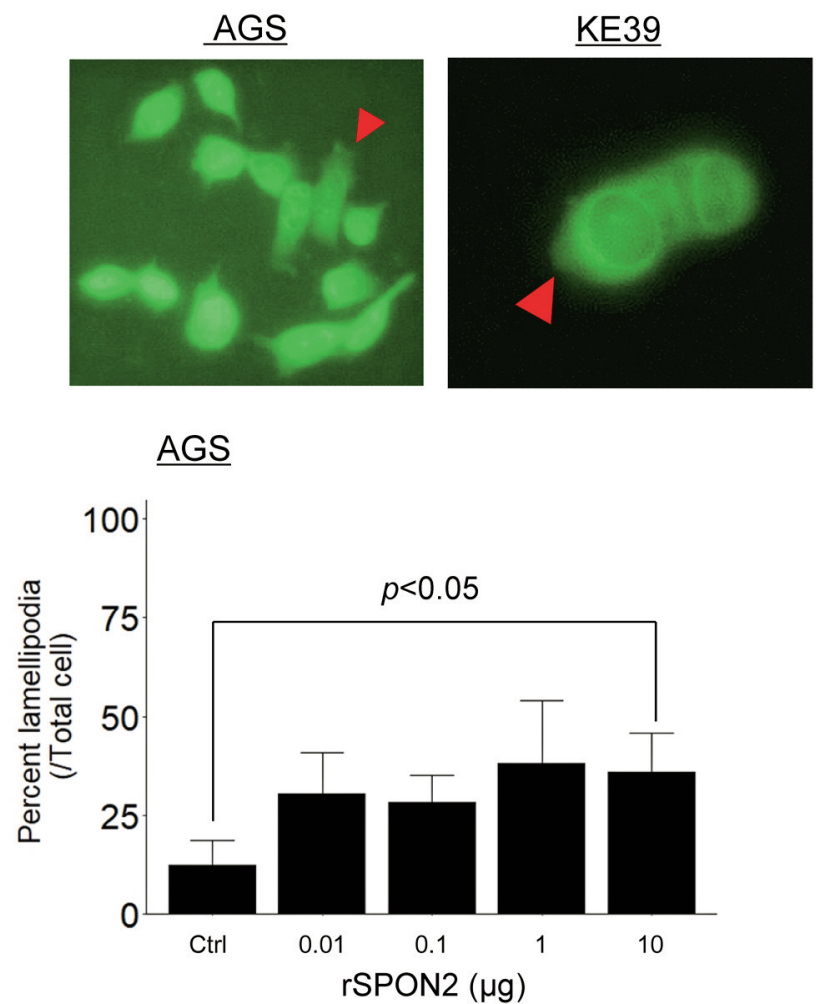

Lamellipodia

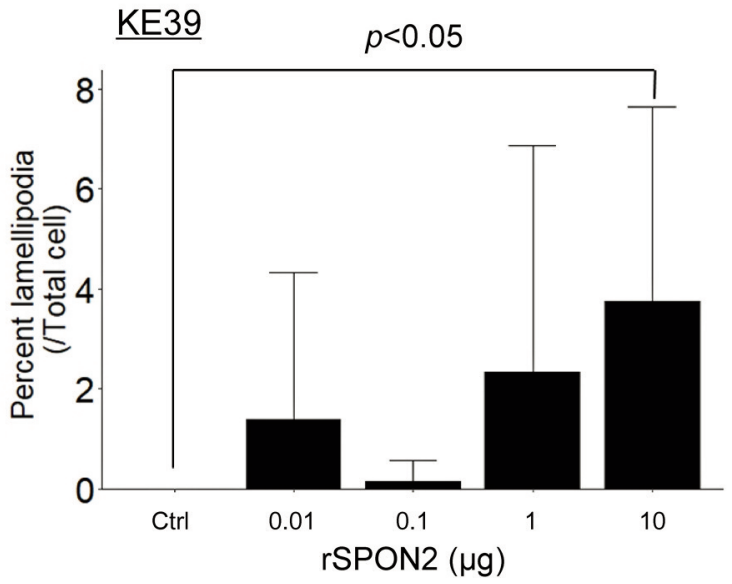

Figure 3. SPON2 can promote the motility of gastric cancer cells. (A) Gene set enrichment analysis (GSEA) of gastric cancer cases using the TCGA dataset. $(B)$ Wound healing migration assay using gastric cancer cells treated with SPON2 recombinant protein ( $r S P O N 2)$. $r S P O N 2$ enhanced the migration capacity of both AGS and KE39 cells (Student's t-test, $p<0.05$ ). (C) Immunofluorescence staining of actin filaments with phalloidin of gastric cancer cells treated with SPON2 recombinant protein (rSPON2). Arrow: lamellipodia. The number of gastric cancer cells with formation of lamellipodia was higher in AGS and KE39 cells treated with rSPON2 than in the control cells (Student's t-test, $p<0.05$ ).

cells in actin cytoskeleton remodeling, immunofluorescence staining of actin filaments was performed in AGS and KE39 cells. The number of cells with formation of lamellipodia, which are thin, sheet-like membrane protrusions found along the leading edge of motile cells (26), was significantly higher in AGS and KE39 cells treated with rSPON2 than in the control cells (Student's $t$-test, $p<0.05$, Figure $3 \mathrm{C}$ ).

\section{Discussion}

Herein, we identified SPON2 as a possible PD-associated gene, and we showed that SPON2 is expressed in CAFs in the TME by, in part, promoting the migration of GC cells. Thus, the expression of SPON2 mRNA in tumor tissues may be a biomarker predicting a poor prognosis of GC patients. CAFs contribute to cancer progression by expressing various factors (e.g., cytokines, chemokines and growth factors such as TGF$\beta$, Interleukin-22, vascular endothelial growth factor and hepatocyte growth factor) that regulate the deposition of extracellular matrix, metabolic reprogramming and tumor cell proliferation, thereby promoting EMT, angiogenesis and chemotherapy resistance in various malignancies including GC $(13,27,28)$. Remarkably, we found that SPON2 was expressed in and secreted from CAFs, and that SPON2 expression was positively associated with the TGF- $\beta$ signaling pathway and EMT according to the GSEA, suggesting that SPON2 affects tumor progression in GC.

SPON2 (also known as mindin) is a member of the mindinF-spondin family of extracellular matrix proteins. Drosophila melanogaster M-spondin, zebrafish mindin 1 and 2 and rat Fspondin and mindin are included in this family (21). A previous study reported that SPON2- $\alpha 4 \beta 1$ integrin signaling activated RhoA and Rac1, increased reorganization of F-actin and facilitated recruitment of M1-macrophage (25), suggesting that SPON2 enhances tumor cell motility by activating RhoA and Rac1 signaling. Herein, we showed that SPON2 enhanced the migration potential and lamellipodia formation of GC cells in vitro. The clinicopathological analysis indicated that high SPON2 expression in GC tissues is positively correlated with tumor size. Furthermore, high expression of SPON2 was associated with a poor prognosis in GC patients. Several lines of evidence also suggest that high expression of SPON2 in 
tumor cells is associated with a poor prognosis in malignancies including colorectal cancer, ovarian cancer and lung cancer (22, 29-31). These findings support our hypothesis that SPON2 facilitates GC progression by promoting GC cell motility.

The development of PD is initiated by the penetration of tumor cells through the gastric wall. Tumor cells need to migrate and invade for successful detachment from the primary tumor and spread into the peritoneal cavity $(7,32)$. SPON2 secreted from CAFs may facilitate PD possibly by enhancing the motility of GC cells. However, it remains unknown how the expression of SPON2 is regulated in CAFs. Further investigation into how SPON2 facilitates PD and the factors that regulate SPON2 expression in CAFs may lead to a good understanding of the association between SPON2 and $\mathrm{PD}$. In vitro co-culture analyses may provide a basis for addressing these challenges as demonstrated elsewhere (33).

In summary, we provide clinical and biological evidence that CAF-derived SPON2 has the potential to promote GC cell motility and potentially PD development, and thus may serve as a biomarker predicting a poor prognosis. There is increasing evidence that CAFs play a critical role in tumor progression and are promising therapeutic targets for malignancies (34). CAF-derived SPON2 might be an attractive therapeutic target to suppress PD in GC.

\section{Conflicts of Interest}

The Authors have no conflicts of interest.

\section{Authors' Contributions}

SK and TM wrote the manuscript. SK and TM conducted the experiments. SK, TM, QH, TT, MY, YT, AK, HO, SI, EO, MM and KM designed the study and interpreted the data. SK and TM prepared the figures and conducted the statistical analysis.

\section{Acknowledgements}

The Authors would like to thank K. Oda, M. Kasagi, S. Sakuma, N. Mishima, T. Kawano, M. Oshiumi and M. Uto for their technical assistance. This work was supported in part by the following grants and foundations: Japan Agency for Medical Research and Development (18ae0101016, 18cm0106504, 18kk0205003 and 18ck0106259), Japan Society for the Promotion of Science Grant-in-Aid for Science Research (20K17556, 20K16416, 20H05039, 20K08930, 20K08960, 19H03715, 19K09176, 19K18057, 18K08649 and 18K15323), JST AIP-PRISM (JPMJCR18Y5) and OITA Cancer Research Foundation.

\section{References}

1 Sung H, Ferlay J, Siegel RL, Laversanne M, Soerjomataram I, Jemal A and Bray F: Global Cancer Statistics 2020: GLOBOCAN estimates of incidence and mortality worldwide for 36 cancers in 185 countries. CA Cancer J Clin 71(3): 209249, 2021. PMID: 33538338. DOI: 10.3322/caac.21660
2 Bando E, Yonemura Y, Takeshita Y, Taniguchi K, Yasui T, Yoshimitsu Y, Fushida S, Fujimura T, Nishimura G and Miwa $\mathrm{K}$ : Intraoperative lavage for cytological examination in 1,297 patients with gastric carcinoma. Am J Surg 178(3): 256-262, 1999. PMID: 10527450. DOI: 10.1016/s0002-9610(99)00162-2

3 Nie RC, Chen S, Yuan SQ, Chen XJ, Chen YM, Zhu BY, Qiu HB, Peng JS and Chen YB: Significant role of palliative gastrectomy in selective gastric cancer patients with peritoneal dissemination: a propensity score matching analysis. Ann Surg Oncol 23(12): 39563963, 2016. PMID: 27380641. DOI: 10.1245/s10434-016-5223-2

4 Bernards N, Creemers GJ, Nieuwenhuijzen GA, Bosscha K, Pruijt JF and Lemmens VE: No improvement in median survival for patients with metastatic gastric cancer despite increased use of chemotherapy. Ann Oncol 24(12): 3056-3060, 2013. PMID: 24121120. DOI: 10.1093/annonc/mdt401

5 Yang XJ, Huang CQ, Suo T, Mei LJ, Yang GL, Cheng FL, Zhou YF, Xiong B, Yonemura Y and Li Y: Cytoreductive surgery and hyperthermic intraperitoneal chemotherapy improves survival of patients with peritoneal carcinomatosis from gastric cancer: final results of a phase III randomized clinical trial. Ann Surg Oncol 18(6): 1575-1581, 2011. PMID: 21431408. DOI: 10.1245/s10434011-1631-5

6 Thomassen I, van Gestel YR, van Ramshorst B, Luyer MD, Bosscha K, Nienhuijs SW, Lemmens VE and de Hingh IH: Peritoneal carcinomatosis of gastric origin: a population-based study on incidence, survival and risk factors. Int J Cancer 134(3): 622-628, 2014. PMID: 23832847. DOI: 10.1002/ijc.28373

7 Kanda M and Kodera Y: Molecular mechanisms of peritoneal dissemination in gastric cancer. World J Gastroenterol 22(30): 68296840, 2016. PMID: 27570420. DOI: 10.3748/wjg.v22.i30.6829

8 Kurashige J, Hasegawa T, Niida A, Sugimachi K, Deng N, Mima K, Uchi R, Sawada G, Takahashi Y, Eguchi H, Inomata M, Kitano S, Fukagawa T, Sasako M, Sasaki H, Sasaki S, Mori M, Yanagihara K, Baba H, Miyano S, Tan P and Mimori K: Integrated molecular profiling of human gastric cancer identifies DDR2 as a potential regulator of peritoneal dissemination. Sci Rep 6: 22371, 2016. PMID: 26934957. DOI: 10.1038/srep22371

$9 \mathrm{Hu}$ Q, Masuda T, Sato K, Tobo T, Nambara S, Kidogami S, Hayashi N, Kuroda Y, Ito S, Eguchi H, Saeki H, Oki E, Maehara $\mathrm{Y}$ and Mimori K: Identification of ARL4C as a peritoneal dissemination-associated gene and its clinical significance in gastric cancer. Ann Surg Oncol 25(3): 745-753, 2018. PMID: 29270876. DOI: 10.1245/s10434-017-6292-6

10 Shimizu D, Saito T, Ito S, Masuda T, Kurashige J, Kuroda Y, Eguchi H, Kodera Y and Mimori K: Overexpression of FGFRI promotes peritoneal dissemination via epithelial-to-mesenchymal transition in gastric cancer. Cancer Genomics Proteomics 15(4): 313-320, 2018. PMID: 29976636. DOI: 10.21873/cgp.20089

11 Shimoda M, Mellody KT and Orimo A: Carcinoma-associated fibroblasts are a rate-limiting determinant for tumour progression. Semin Cell Dev Biol 21(1): 19-25, 2010. PMID: 19857592. DOI: $10.1016 /$ j.semcdb.2009.10.002

12 Kalluri R: The biology and function of fibroblasts in cancer. Nat Rev Cancer 16(9): 582-598, 2016. PMID: 27550820. DOI: $10.1038 /$ nrc. 2016.73

13 Kalluri R and Zeisberg M: Fibroblasts in cancer. Nat Rev Cancer 6(5): 392-401, 2006. PMID: 16572188. DOI: 10.1038/nrc1877

14 Zeisberg EM, Potenta S, Xie L, Zeisberg M and Kalluri R: Discovery of endothelial to mesenchymal transition as a source for carcinoma-associated fibroblasts. Cancer Res 67(21): 10123- 
10128, 2007. PMID: 17974953. DOI: 10.1158/0008-5472.CAN07-3127

15 Pietras K and Ostman A: Hallmarks of cancer: interactions with the tumor stroma. Exp Cell Res 316(8): 1324-1331, 2010. PMID: 20211171. DOI: 10.1016/j.yexcr.2010.02.045

16 Kitagawa A, Masuda T, Takahashi J, Tobo T, Noda M, Kuroda Y, Hu Q, Kouyama Y, Kobayashi Y, Kuramitsu S, Sato K, Fujii A, Yoshikawa Y, Wakiyama H, Shimizu D, Tsuruda Y, Eguchi H, Doki Y, Mori M and Mimori K: KIF15 expression in tumorassociated monocytes is a prognostic biomarker in hepatocellular carcinoma. Cancer Genomics Proteomics 17(2): 141-149, 2020. PMID: 32108036. DOI: 10.21873/cgp.20174

17 Hasegawa T, Yashiro M, Nishii T, Matsuoka J, Fuyuhiro Y, Morisaki T, Fukuoka T, Shimizu K, Shimizu T, Miwa A and Hirakawa K: Cancer-associated fibroblasts might sustain the stemness of scirrhous gastric cancer cells via transforming growth factor- $\beta$ signaling. Int J Cancer 134(8): 1785-1795, 2014 PMID: 24155219. DOI: $10.1002 / \mathrm{ijc} .28520$

18 Masuda TA, Inoue H, Nishida K, Sonoda H, Yoshikawa Y, Kakeji Y, Utsunomiya T and Mori M: Cyclin-dependent kinase 1 gene expression is associated with poor prognosis in gastric carcinoma. Clin Cancer Res 9(15): 5693-5698, 2003. PMID: 14654553.

19 Wakiyama H, Masuda T, Motomura Y, Hu Q, Tobo T, Eguchi H, Sakamoto K, Hirakawa M, Honda H and Mimori K: Cytolytic activity (CYT) score is a prognostic biomarker reflecting host immune status in hepatocellular carcinoma (HCC). Anticancer Res 38(12): 6631-6638, 2018. PMID: 30504371. DOI: 10.21873/anticanres. 13030

20 Mizuno H, Kitada K, Nakai K and Sarai A: PrognoScan: a new database for meta-analysis of the prognostic value of genes. BMC Med Genomics 2: 18, 2009. PMID: 19393097. DOI: 10.1186/1755-8794-2-18

21 Feinstein Y, Borrell V, Garcia C, Burstyn-Cohen T, Tzarfaty V, Frumkin A, Nose A, Okamoto H, Higashijima S, Soriano E and Klar A: F-spondin and mindin: two structurally and functionally related genes expressed in the hippocampus that promote outgrowth of embryonic hippocampal neurons. Development 126(16): 3637-3648, 1999. PMID: 10409509.

22 Lu H, Feng Y, Hu Y, Guo Y, Liu Y, Mao Q and Xue W: Spondin 2 promotes the proliferation, migration and invasion of gastric cancer cells. J Cell Mol Med 24(1): 98-113, 2020. PMID: 31691494. DOI: $10.1111 / \mathrm{jcmm} .14618$

23 Calon A, Tauriello DV and Batlle E: TGF-beta in CAF-mediated tumor growth and metastasis. Semin Cancer Biol 25: 15-22, 2014. PMID: 24412104. DOI: 10.1016/j.semcancer.2013.12.008

24 Sun F, Feng M and Guan W: Mechanisms of peritoneal dissemination in gastric cancer. Oncol Lett 14(6): 6991-6998, 2017. PMID: 29344127. DOI: 10.3892/ol.2017.7149

25 Zhang YL, Li Q, Yang XM, Fang F, Li J, Wang YH, Yang Q, Zhu L, Nie HZ, Zhang XL, Feng MX, Jiang SH, Tian GA, Hu LP, Lee HY, Lee SJ, Xia Q and Zhang ZG: SPON2 promotes M1-like macrophage recruitment and inhibits hepatocellular carcinoma metastasis by distinct integrin-Rho GTPase-hippo pathways. Cancer Res 78(9): 2305-2317, 2018. PMID: 29440144. DOI: 10.1158/0008-5472.CAN-17-2867
26 Bodor DL, Pönisch W, Endres RG and Paluch EK: Of cell shapes and motion: The physical basis of animal cell migration. Dev Cell 52(5): 550-562, 2020. PMID: 32155438. DOI: 10.1016/j.devce1.2020.02.013

27 Yan Y, Wang LF and Wang RF: Role of cancer-associated fibroblasts in invasion and metastasis of gastric cancer. World J Gastroenterol 21(33): 9717-9726, 2015. PMID: 26361418. DOI: 10.3748/wjg.v21.i33.9717

28 Miki Y, Yashiro M, Moyano-Galceran L, Sugimoto A, Ohira M and Lehti K: Crosstalk between cancer associated fibroblasts and cancer cells in scirrhous type gastric cancer. Front Oncol 10: 568557, 2020. PMID: 33178597. DOI: 10.3389/fonc.2020.568557

29 Zhou Q, Li L, Zhao B and Guan KL: The hippo pathway in heart development, regeneration, and diseases. Circ Res 116(8): 1431-1447, 2015. PMID: 25858067. DOI: 10.1161/ CIRCRESAHA.116.303311

30 Simon I, Liu Y, Krall KL, Urban N, Wolfert RL, Kim NW and McIntosh MW: Evaluation of the novel serum markers B7-H4, Spondin 2, and DcR3 for diagnosis and early detection of ovarian cancer. Gynecol Oncol 106(1): 112-118, 2007. PMID: 17490732. DOI: 10.1016/j.ygyno.2007.03.007

31 Yuan X, Bian T, Liu J, Ke H, Feng J, Zhang Q, Qian L, Li X, Liu Y and Zhang J: Spondin2 is a new prognostic biomarker for lung adenocarcinoma. Oncotarget 8(35): 59324-59332, 2017. PMID: 28938639. DOI: 10.18632/oncotarget.19577

$32 \mathrm{Hu}$ Q, Ito S, Yanagihara K and Mimori K: Molecular mechanism of peritoneal dissemination in gastric cancer. Journal of Cancer Metastasis and Treatment 4(7): 39, 2018. DOI: 10.20517/23944722.2018.08

33 Musa M, Ouaret D and Bodmer WF: In vitro analyses of interactions between colonic myofibroblasts and colorectal cancer cells for anticancer study. Anticancer Res 40(11): 60636073, 2020. PMID: 33109544. DOI: 10.21873/anticanres.14627

34 Su S, Chen J, Yao H, Liu J, Yu S, Lao L, Wang M, Luo M, Xing Y, Chen F, Huang D, Zhao J, Yang L, Liao D, Su F, Li M, Liu $\mathrm{Q}$ and Song E: CD $10^{+} \mathrm{GPR} 77^{+}$cancer-associated fibroblasts promote cancer formation and chemoresistance by sustaining cancer stemness. Cell 172(4): 841-856.e16, 2018. PMID: 29395328. DOI: 10.1016/j.cell.2018.01.009
Received April 18, 2021

Revised May 14, 2021

Accepted May 26, 2021 